\title{
Entomological Investigation Detects Dengue Virus Type 1 in Aedes (Stegomyia) albopictus (Skuse) during the 2015-16 Outbreak in Hawaii
}

\author{
Jeomhee M. Hasty, ${ }_{1}^{1}$ Gilberto E. Felix, ${ }^{2}$ Manuel Amador, ${ }^{2}$ Roberto Barrera, ${ }^{2}$ Gilberto S. Santiago, ${ }^{2}$ Lynn Nakasone, ${ }^{1}$ Sarah Y. Park, ${ }^{1}$ \\ Steven Okoji, ${ }^{1}$ Eric Honda, ${ }^{1}$ Bernard Asuncion, ${ }^{1}$ Maricia Save, ${ }^{1}$ Jorge L. Munoz-Jordan, ${ }^{2}$ Stephanie Martinez-Conde, ${ }^{2}$ \\ Freddy A. Medina, ${ }^{2}$ Stephen H. Waterman, ${ }^{2}$ Lyle R. Petersen, ${ }^{3}$ David I. Johnston, ${ }^{1}$ and Ryan R. Hemme ${ }^{2 *}$ \\ ${ }^{1}$ Hawaii State Department of Health, Honolulu, Hawaii; ${ }^{2}$ Division of Vector-Borne Diseases, Centers for Disease Control and Prevention, Dengue \\ Branch, San Juan, Puerto Rico; ${ }^{3}$ Division of Vector-Borne Diseases, Centers for Disease Control and Prevention, Fort Collins, Colorado
}

\begin{abstract}
A dengue outbreak occurred on Hawaii Island between September 2015 and March 2016. Entomological investigations were undertaken between December 2015 and February 2016 to determine which Aedes mosquito species were responsible for the outbreak. A total of 3,259 mosquitoes were collected using a combination of CDC autocidal gravid ovitraps, Biogents BG-Sentinel traps, and hand-nets; immature mosquitoes were collected during environmental surveys. The composition of species was Aedes albopictus (58\%), Aedes aegypti (25\%), Wyeomyia mitchelli (7\%), Aedes vexans (5\%), Culex quinquefasciatus (4\%), and Aedes japonicus (1\%). Adult mosquitoes were analyzed by real-time reverse transcription polymerase chain reaction (PCR) for the presence of dengue virus (DENV) RNA. Of the 185 pools of female mosquitoes tested, 15 containing $A$ e. albopictus were positive for the presence of DENV type 1 RNA. No virus was detected in pools of the remaining species. Phylogenetic analysis showed the virus strain belonged to genotype I and was closely related to strains that were circulating in the Pacific between 2008 and 2014. This is the first report of detection of DENV in Ae. albopictus from Hawaii.
\end{abstract}

\section{INTRODUCTION}

Dengue is a vector-borne disease caused by four types of dengue viruses (DENV1-4, Flaviviridae, and Flavivirus) and is transmitted by the bite of infected Aedes mosquitoes, primarily Aedes aegypti and Aedes albopictus. The global burden of dengue is estimated as 390 million infections per year which is larger than previously believed. ${ }^{1}$ The rise in dengue incidence can be attributed to geographic range expansion of competent vectors, increased speed at which viremic humans can transport viruses across large distances, and increased urbanization. $^{2}$

In October 2015, the Hawaii State Department of Health $(\mathrm{HDOH})$ was notified of a resident who tested positive for dengue IgM and had no history of travel outside of Hawaii Island. ${ }^{3}$ Interviews indicated that contact with mosquitoes most likely occurred on the western side of Hawaii Island near Kona, Hawaii, and marked the first recorded case of a locally acquired infection since the 2011 outbreak on Oahu., ${ }^{3,4}$ The outbreak spread along populated coastal areas on the western side of the island near Kailua-Kona, Captain Cook, and Milolii, and at its peak cases were reported across the island, including Oceanview, Waipio, Hilo, and Puna (Figure 1). By the end of the outbreak in March 2016, 264 dengue cases were reported to the $\mathrm{HDOH}$.

In the late 1890s, illnesses with dengue-like symptoms were reported after Aedes mosquitoes were introduced to Hawaii. ${ }^{5,6}$ Subsequent dengue outbreaks were recorded in 1903, 1912, 1943-1945, 2001, and 2011. ${ }^{3,5-9}$ Aedes aegypti was the primary vector of dengue on Hawaii during the 1943-1945 Honolulu dengue epidemic. ${ }^{8}$ In response, Ae. aegypti was eliminated from Oahu making Ae. albopictus the most prevalent Aedes species in the state. ${ }^{5,8,10-13}$ As part of the elimination campaign, a statewide survey

\footnotetext{
${ }^{*}$ Address correspondence to Ryan R. Hemme, Entomology and Ecology Activity, Dengue Branch, Centers for Disease Control and Prevention, 1324 Calle Cañada, San Juan 00920, Puerto Rico. E-mail: rhemme@cdc.gov
}

conducted in the 1960s found Ae. aegypti had been reduced on Hawaii Island. ${ }^{5,8,10,13-16}$ The 2001 dengue outbreak, caused by DENV-1, was the first identified autochthonous transmission of DENV in the state in 56 years. ${ }^{7}$ Entomological surveys at the time found Ae. albopictus in $29 \mathrm{com}-$ munities located on Oahu, Maui, Hawaii Island, Molokai, and Kauai and found Ae. aegypti on Hawaii Island. ${ }^{7}$ No DENVpositive mosquito pools were detected. ${ }^{7}$

Dengue epidemics vectored by Ae. albopictus are less common than those vectored by $A e$. aegypti because presumably $A$ e. aegypti is better adapted to urban environments, exploits artificial containers for oviposition, and is highly anthropophagic, preferring to feed almost exclusively on humans. ${ }^{17-19}$ By contrast, Ae. albopictus will opportunistically feed on numerous mammals, including humans, dogs, cattle, and swine. ${ }^{20}$ Aedes albopictus is more common in forested or rural areas, and it will oviposit in artificial or natural (tree holes) containers when available. ${ }^{21}$ The objectives of this investigation were to determine the respective roles of $A e$. aegypti and $A e$. albopictus in the 2015-16 dengue outbreak and document the spatial distribution of these species on Hawaii Island.

\section{MATERIALS AND METHODS}

Hawaii Island is the largest island in the State of Hawaii. Seasons are divided into summer, occurring between May and October and winter from November to April, with the winter season receiving more precipitation. The north and east side of the island is generally wetter and more humid because of orographic precipitation. This investigation took place between mid-November 2015 and midFebruary 2016. Most of the mosquito trapping occurred on the western side of Hawaii Island, between Kona and Milolii, and additional collections were conducted in Waipio, Oceanview, Hilo, and Puna (Figure 1, Table 1). The locations sampled for this investigation were residential neighborhoods, including rural and sylvan areas within $800 \mathrm{~m}$ of clusters of laboratory-confirmed DENV cases 


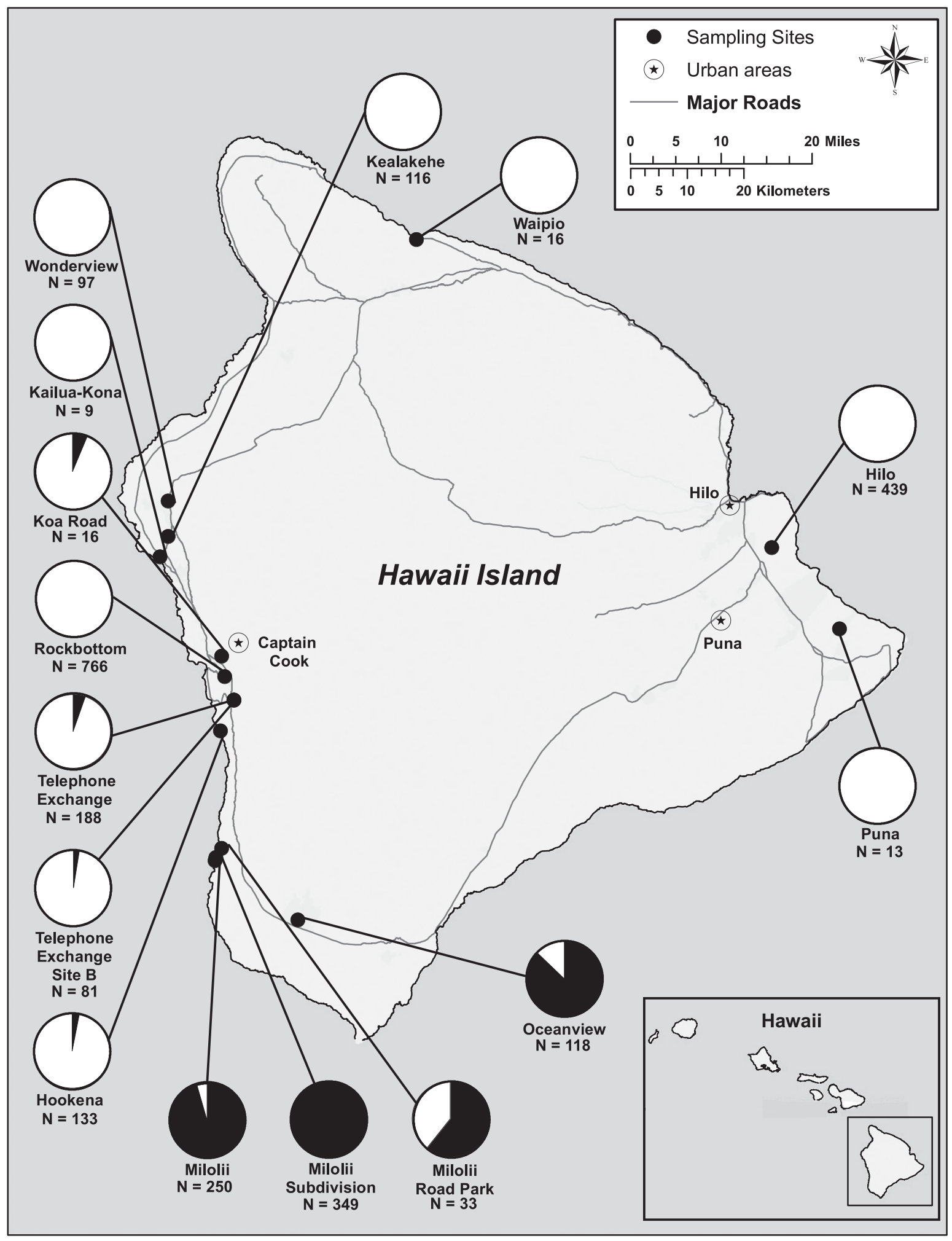

FIGURE 1. Locations and combined total Aedes aegypti and Aedes albopictus collected at each sampling site on Hawaii Island, 2015-2016. Black indicates the proportion of $A e$. aegypti and white indicates the proportion of Aedes albopictus. This figure appears in color at www.ajtmh.org.

(Tables 1 and 2). Rock Bottom was relatively rural and surrounded by macadamia nut orchards and forest compared with Wonderview and Kealakehe, which were residential neighborhoods near Kona. Hookena was a State Park on the western side of the island and Waipio was a rural community composed of single-family houses surrounded by forest. The village of Milolii, Milolii subdivision, and Oceanview were built on lava beds, did not have access to municipal water, and thus, stored water in cisterns or water storage drums/containers. 
TABLE 1

Mosquito species collected using autocidal gravid ovitraps (AGOs), Biogents BG-Sentinel Traps (BGs), hand-nets (HNs), and larval collections (LCs) from sampling sites on the Big Island of Hawaii during the 2015-2016 dengue virus outbreak

\begin{tabular}{|c|c|c|c|c|c|c|c|c|c|c|c|c|c|c|c|}
\hline \multirow[b]{2}{*}{ Sampling site } & \multirow[b]{2}{*}{ Trap } & \multirow{2}{*}{$\begin{array}{l}\text { Number } \\
\text { of traps }\end{array}$} & \multirow[b]{2}{*}{ Date } & \multicolumn{2}{|c|}{ Aedes aegypti } & \multicolumn{2}{|c|}{$\begin{array}{c}\text { Ae. } \\
\text { albopictus }\end{array}$} & \multicolumn{2}{|c|}{$\begin{array}{c}\text { Aedes } \\
\text { japonicus }\end{array}$} & \multicolumn{2}{|c|}{$\begin{array}{l}\text { Aedes } \\
\text { vexans }\end{array}$} & \multicolumn{2}{|c|}{$\begin{array}{c}\text { Culex } \\
\text { quinquefasciatus }\end{array}$} & \multicolumn{2}{|c|}{$\begin{array}{c}\text { Wyeomyia } \\
\text { mitchelli }\end{array}$} \\
\hline & & & & Female & Male & Female & Male & Female & Male & Female & Male & Female & Male & Female & Male \\
\hline \multirow[t]{3}{*}{ Hookena } & $\mathrm{BG}^{\mathrm{C}}$ & 2 & November 17-19, 2015 & $3^{*}$ & 1 & $40 \dagger$ & 15 & 0 & 0 & 0 & 0 & 0 & 0 & 0 & 0 \\
\hline & $\mathrm{BG}^{\mathrm{NC}}$ & 3 & December 7-12, 2015 & 0 & 0 & 22 & 9 & 0 & 0 & 0 & 0 & 0 & 1 & 0 & 0 \\
\hline & $\mathrm{BG}^{\mathrm{C}}$ & 4 & December 13, 2015 & 0 & 0 & $32 \dagger$ & 11 & 0 & 0 & 0 & 0 & 0 & 0 & 0 & 0 \\
\hline Hilo & $\mathrm{BG}^{\mathrm{C}}$ & 6 & December 10-17, 2015 & 0 & 0 & $359^{*}$ & 80 & 0 & 0 & 150 & 0 & 11 & 0 & 0 & 0 \\
\hline Kailua-Kona & $\mathrm{BG}^{\mathrm{C}}$ & 3 & February $11-13,2016$ & 0 & 0 & $9^{*}$ & 0 & 0 & 0 & 0 & 0 & 0 & 2 & 0 & 0 \\
\hline \multirow[t]{2}{*}{ Kealakehe } & $\mathrm{BG}^{\mathrm{BL}}$ & 20 & $\begin{array}{l}\text { January 21-February 4, } \\
2016\end{array}$ & 0 & 0 & $4^{*}$ & 1 & 0 & 0 & 0 & 0 & 17 & 1 & 0 & 0 \\
\hline & AGO & 20 & $\begin{array}{l}\text { January 21-February 4, } \\
2016\end{array}$ & 0 & 0 & $78^{\star}$ & 33 & 0 & 0 & 0 & 0 & 9 & 1 & 0 & 0 \\
\hline Koa Road & $B G^{C}$ & 2 & December 19-21, 2015 & $1^{*}$ & 0 & 12 & 3 & 0 & 0 & 0 & 0 & 0 & 0 & 0 & 0 \\
\hline Milolii & $\mathrm{BG}^{\mathrm{C}}$ & 6 & January 5-10, 2016 & $186^{\star}$ & 53 & $10^{*}$ & 1 & 0 & 0 & 0 & 0 & 6 & 3 & 0 & 0 \\
\hline Milolii Road & $B G^{C}$ & 3 & December 22-23, 2015 & $47^{\star}$ & 48 & $7^{\star}$ & 3 & 0 & 0 & 0 & 0 & 0 & 0 & 0 & 0 \\
\hline Milolii Road Park & $B G^{C}$ & 3 & January 4-10, 2016 & 19 & 1 & 13 & 0 & 0 & 0 & 0 & 0 & 1 & 0 & 0 & 0 \\
\hline $\begin{array}{l}\text { Milolii } \\
\quad \text { Subdivision }\end{array}$ & $B G^{C}$ & 3 & January 4-10, 2016 & 260 & 89 & 0 & 0 & 0 & 0 & 0 & 0 & 0 & 0 & 0 & 0 \\
\hline Oceanview & LC & - & January 29, 2016 & 51 & 52 & 5 & 10 & 0 & 0 & 0 & 0 & 0 & 0 & 0 & 0 \\
\hline Puna & $B G^{C}$ & 4 & December 8-9, 2015 & 0 & 0 & $9^{*}$ & 4 & 0 & 0 & 0 & 0 & 9 & 0 & 0 & 0 \\
\hline \multirow[t]{2}{*}{ Rock Bottom } & $\mathrm{BG}^{\mathrm{BL}}$ & 20 & December 3-17, 2015 & 0 & 0 & $34^{*}$ & 14 & 0 & 0 & 0 & 0 & 5 & 0 & 0 & 0 \\
\hline & AGO & 20 & December 3-17, 2015 & 0 & 0 & $473^{*}$ & 245 & $5^{\star}$ & 0 & 0 & 0 & 25 & 6 & 193 & 0 \\
\hline \multirow{5}{*}{$\begin{array}{l}\text { Telephone } \\
\text { Exchange }\end{array}$} & $B G^{N C}$ & 1 & November 17, 2015 & $1^{*}$ & 0 & 0 & 1 & 0 & 0 & 0 & 0 & 0 & 0 & 0 & 0 \\
\hline & $\mathrm{BG}^{\mathrm{C}}$ & 1 & December 3-5, 2015 & $1^{*}$ & 0 & 30 & 8 & 3 & 0 & 0 & 0 & 0 & 0 & 1 & 0 \\
\hline & $\mathrm{BG}^{\mathrm{NC}}$ & 1 & December 5-11, 2015 & 0 & 0 & 24 & 11 & 0 & 0 & 0 & 0 & 0 & 0 & 0 & 0 \\
\hline & $\mathrm{BG}^{\mathrm{C}}$ & 1 & December 13, 2015 & 0 & 0 & 2 & 3 & 0 & 0 & 0 & 0 & 0 & 0 & 0 & 0 \\
\hline & $\mathrm{BG}^{\mathrm{C}}$ & 6 & December 15-20, 2015 & $10^{*}$ & 0 & $149^{\star}$ & 29 & $15^{\star}$ & 0 & 0 & 0 & 2 & 0 & 40 & 0 \\
\hline Waipio & $\mathrm{HN}$ & - & January 26, 2016 & 0 & 0 & $12^{*}$ & 4 & 0 & 0 & 0 & 0 & 0 & 0 & 0 & 0 \\
\hline \multirow[t]{2}{*}{ Wonderview } & $B G^{B L}$ & 20 & $\begin{array}{l}\text { January 21-February 4, } \\
2016\end{array}$ & 0 & 0 & $5^{\star}$ & 1 & 0 & 0 & 0 & 0 & 12 & 0 & 0 & 0 \\
\hline & AGO & 20 & $\begin{array}{l}\text { January 21-February 4, } \\
2016\end{array}$ & 0 & 0 & $83^{*}$ & 8 & 0 & 0 & 0 & 0 & 6 & 5 & 1 & 0 \\
\hline TOTAL & - & - & - & 579 & 244 & 1,412 & 494 & 23 & 0 & 150 & 0 & 103 & 19 & 235 & 0 \\
\hline
\end{tabular}

$\mathrm{BG}^{\mathrm{C}}$ indicates trap was baited with carbon dioxide, $\mathrm{BG}^{\mathrm{NC}}$ indicates no carbon dioxide was used, $\mathrm{BG} \mathrm{BL}^{\mathrm{BL}}$ indicates that a black covering was used as an visual attractant, $\mathrm{LC}$ indicates specimens were collected during an immature collection, and $\mathrm{HN}$ indicates specimens were collected with $\mathrm{HN}$ for viral RNA detection.

* Indicates specimens were tested for viral RNA.

$\dagger$ Indicates not all of the specimens collected at this site were tested for viral RNA.

At Kealakehe, Rock Bottom, and Wonderview, 20 CDC autocidal gravid ovitraps (AGO) and 20 Biogents BG-Sentinel (BG) traps with black covers and without carbon dioxide $\left(\mathrm{CO}_{2}\right)$ were paired and placed on opposite sides of houses within a 200-m radius of the case-patient (Table 1). ${ }^{22}$ Trapping at the three locations was conducted for 14 days. At Hilo, Telephone Exchange, and Milolii, BG traps using $\mathrm{CO}_{2}$ lures without black covers were deployed for 7 days (Table 1 ). BG-Sentinel traps were monitored daily and AGO traps were checked every 3-4 days. Additional BG traps without black covers that varied in use of $\mathrm{CO}_{2}$ were placed in areas where DENV or the presence of Ae. aegypti was suspected, and informal surveys of aquatic habitats were performed at Waipio and Oceanview (Table 1).

Mosquito collections were transported on ice to the laboratory and were killed by freezing. All mosquitoes were sexed and identified to species. Female Ae. aegypti, Ae. albopictus, and Aedes japonicus were placed in 2-mL vials containing not more than 10 female mosquitoes and stored at $-20^{\circ} \mathrm{C}$ until they could be shipped to the CDC Dengue Branch where they were stored at $-80^{\circ} \mathrm{C}$ until tested for viral RNA.

Mosquito pools were suspended in tissue culture media and macerated using the Qiagen TissueLyser II instrument. Viral RNA was extracted and detected by dengue type-specific real-time reverse transcription PCR as described by Santiago et al. ${ }^{23}$ PCR reactions with cycle threshold values below 37 were considered positive. Maximum likelihood estimates of the minimum infection rate for Ae. albopictus were calculated using PooledlnfRate version 4.0 (Fort Collins, CO). ${ }^{24}$

The envelope glycoprotein coding region (E gene) of DENV-1 was sequenced directly from the mosquito pool macerates and nine human clinical serum specimens that tested positive by PCR. The target region was amplified using serotype-specific primers and the resulting amplicon $(1,743 \mathrm{bp})$ was sequenced using the Sanger bidirectional method from eight bidirectional and overlapping sequencing reactions using Applied BioSystems (Foster City, CA) BigDye Terminator v. 3.1 sequencing kits. Sequences were obtained directly from the original samples; virus isolation was not attempted. A total of 29 DENV-1 E gene sequences were obtained, including from $12 \mathrm{Ae}$. albopictus pools collected during the 2015 outbreak, nine clinical serum specimens from symptomatic humans collected in the same region during the same outbreak by the $\mathrm{HDOH}$, and eight clinical serum specimens from symptomatic humans collected during the 2001 DENV-1 outbreak. ${ }^{7}$ To understand the genetic relatedness of these sequences in context with the South Pacific, we reconstructed a Bayesian maximum clade credibility phylogenetic tree with the 29 sequences obtained from this study and 42 sequences obtained from GenBank using BEAST v. 1.8.4. Parameters for BEAST (Edinburgh, UK) include 30 million MCMC, time of the most recent common ancestor for the 2015 monophyletic lineage, and a strict molecular clock to achieve acceptable statistical values (effective sample size $>200$ ). 
TABLE 2

Mean female captures (autocidal gravid ovitrap [AGO], trap-week; BG-Sentinel [BG] trap-day), number of pools tested, pools positive for dengue virus (DENV) RNA, and DENV infection rates from three sampling sites in Hawaii Island using AGOs and Biogents BG traps without carbon dioxide for 14 trap-nights between December 2015 and February 2016

\begin{tabular}{|c|c|c|c|c|c|c|c|c|}
\hline \multirow[b]{2}{*}{ Sampling site } & \multicolumn{2}{|c|}{ Mean female Aedes albopictus } & \multicolumn{2}{|c|}{$\begin{array}{c}\text { Number of pools } \\
\text { tested (average size) }\end{array}$} & \multicolumn{2}{|c|}{$\begin{array}{l}\text { DENV-positive } \\
\text { pools (\%) }\end{array}$} & \multicolumn{2}{|c|}{ DENV infection rate per $1,000(95 \% \mathrm{Cl})$} \\
\hline & AGO (trap-week) & BG (trap-day) & AGO & $B G$ & AGO & $B G$ & AGO & $B G$ \\
\hline Kealakehe & $0.1(0.0-0.2)$ & $0.3(0.2-0.4)$ & $2(1)$ & $15(5)$ & 0 & 0 & - & - \\
\hline Rock Bottom & $0.9(0.4-1.3)$ & $1.6(1.4-1.9)$ & $7(5)$ & $62(8)$ & $2(29)$ & $13(21)$ & $62.2(12.3,202.4)$ & $30.4(17.2,50.2)$ \\
\hline Wonderview & $0.1(0.1-0.2)$ & $0.3(0.3-0.4)$ & $3(2)$ & $14(6)$ & 0 & 0 & - & - \\
\hline
\end{tabular}

\section{RESULTS}

We collected six species of mosquito from three genera; the most common mosquito species collected was Ae. albopictus, followed by Ae. aegypti, Wyeomyia mitchelli, Aedes vexans, Culex quinquefasciatus, and Ae. japonicus (Table 1). Aedes albopictus was collected at all 15 sampled sites and was the only or most abundant species at 12 of the sites, whereas Ae. aegypti was found at five sites and was most abundant on the southwest side of Hawaii Island near the Milolii and Oceanview sites (Figure 1).

In total, we screened 185 pools of female mosquitoes for the presence of DENV RNA of which 135 were Ae. albopictus, 38 were $A$ e. aegypti, and 12 were Ae. japonicus. We detected viral RNA for DENV-1 in 15 pools of $A$ e. albopictus from Rock Bottom, two of which were from pools using AGOs and 13 were pools from BG traps. Infection rates at Rock Bottom were 62.2 per 1,000 and 30.4 per 1,000 for $A G O$ and $B G$ traps, respectively (Table 2). No virus-positive pools were detected in pools of Ae. aegypti or Ae. albopictus collected in Telephone Exchange, Hilo, or Milolii (Table 3).

In general, all the 21 sequences obtained from the 2015 outbreak grouped together as a monophyletic lineage and belonged to genotype I, including those derived from mosquito macerates and human specimens (Figure 2, Supplemental Table 1). No phylogenetic difference was detected between mosquito and human host sequences. Two 2015 sequences grouped separately: sequence US/ DB207/2015 grouped together with a 2013 Australian (Cairns) sequence and sequence US/DB203/2014 grouped with a cluster of sequences that circulated in Australia and Papua New Guinea between 2008 and 2014. Time of the most recent common ancestor suggests that the Hawaiian 2015 lineage emerged in the region by 2013 (1.4-3.36 years $95 \%$ highest posterior density) and is closely related to sequences from New Caledonia with contemporary circulation. However, all eight sequences from 2001 grouped separately and belonged to genotype IV.

\section{DISCUSSION}

During the 2015-16 DENV outbreak on Hawaii Island, Ae. albopictus was found at all sampled sites where laboratoryconfirmed case-patients resided, and RNA of DENV-1 was detected by RT-PCR from pools of Ae. albopictus collected at Rock Bottom. Phylogenetic analysis showed the virus identified from Ae. albopictus and human specimens belonged to genotype I and were monophyletic. In addition, extensive mosquito trapping near clusters of DENV-confirmed casepatients in Wonderview, Kealakehe, and Hilo detected Ae. albopictus, but no $A$ e. aegypti. These results indicated that $A e$. albopictus was the primary vector responsible for transmitting DENV on Hawaii Island during the 2015-16 outbreak. This is the first entomological investigation to detect DENV in Ae. albopictus in the state of Hawaii, confirming its importance in DENV transmission on the islands of Hawaii.

We are unable to discount the possibility that $A$ e. aegypti was involved at some foci. Aedes aegypti was found at six sites where dengue case-patients resided, three of which were clustered near the town of Milolii. Most mosquitoes collected at those locations (94\%) were Ae. aegypti $(N=747$; Figure 1). It is significant that Ae. aegypti was dominant in communities that relied on water storage for domestic use and for drinking. Furthermore, entomological investigations collected adult Ae. aegypti and Ae. albopictus in Hookena Beach Park, which was identified as a possible site of exposure during the early phases of the outbreak. ${ }^{3}$ Not all mosquito specimens were tested for viral RNA, as those collected in neighborhoods where no recent DENV cases were reported would have been less likely to be infected with DENV. Consequently, we may have not detected some virus-positive specimens because of timing of the investigation. Furthermore, sampling at Rock Bottom was conducted in December, concurrent with active transmission in humans, whereas in Kealakehe and Wonderview, specimens were collected in January, 1-2 weeks after the case-patients were reported to the $\mathrm{HDOH}$. Finally, not all our investigations could be completed before vector control activities were conducted in

TABLE 3

Mean female captures per trap-day, number of pools tested, and pools positive for dengue virus (DENV) RNA at sampling sites in Hawaii Island using Biogents BG-Sentinel traps baited carbon dioxide for 7 trap-nights between December 2015 and January 2016

\begin{tabular}{|c|c|c|c|c|c|c|}
\hline \multirow[b]{2}{*}{ Sampling site } & \multicolumn{2}{|c|}{ Mean female per trap-day } & \multicolumn{2}{|c|}{ Number of pools tested (avg. size) } & \multicolumn{2}{|c|}{ DENV-positive pools (\%) } \\
\hline & Ae. aegypti & Ae. albopictus & Ae. aegypti & Ae. albopictus & Ae. aegypti & Ae. albopictus \\
\hline Telephone Exchange & $0.2(0.1-0.1)$ & $3.4(2.5-4.6)$ & $6(2)$ & $16(10)$ & 0 & 0 \\
\hline Hilo & 0 & $8.6(4.6-12.6)$ & 0 & $38(9)$ & 0 & 0 \\
\hline Milolii & $4.4(3.0-5.8)$ & $0.2(0.1-0.5)$ & $22(8)$ & $5(2)$ & 0 & 0 \\
\hline
\end{tabular}

Ae. aegypt $=$ Aedes aegypt $;$ Ae. albopictus = Aedes albopictus 




FIGURE 2. Genetic relatedness of Hawaii dengue virus (DENV-1) strains. Bayesian phylogenetic reconstruction using a maximum clade credibility tree that includes 71 DENV-1 E gene $(1,485 \mathrm{bp})$ sequences and corresponds to the complete gene sequence. Posterior probabilities of major tree nodes are shown. (*) next to taxon label indicates the sequence obtained during the 2015-2016 dengue outbreak on Hawaii Island. Sequences without $\left(^{*}\right)$ were obtained from GenBank. Mosquito icon next to taxa label indicates sequences derived from Aedes albopictus hosts. Sequences without the mosquito icon were derived from human hosts. 
Milolii, Telephone Exchange, and Hookena, which might have affected our findings.

Aedes albopictus was the vector responsible for transmitting virus in the 2001 and 2015 dengue outbreaks in Hawaii, and the outbreaks were caused by separate virus introductions (Figure 2). It has been suggested that DENV outbreaks transmitted by Ae. albopictus are less explosive and tend to smolder as was observed during the 2015 outbreak. ${ }^{17,25,26}$ However, reports of dengue outbreaks in China show that Ae. albopictus can drive large epidemics, suggesting that outcomes of outbreaks are in part a result of complex interactions between mosquito and human behaviors, and demographics. ${ }^{27-31}$ For example, during this outbreak on Hawaii Island, dengue transmission might have been slowed because of host feeding behaviors of Ae. albopictus (wide range of hosts, exophagy) and low population density that limited human-mosquito interactions compared with areas with Ae. aegypti or higher densities of humans. ${ }^{31}$ Recent population growth, demographic shifts to urban areas, and its prominence as an important destination and hub in the Pacific for civilians and the U.S. military may alter the epidemiology of future outbreaks. ${ }^{27,32-34}$ The presence of two competent vectors and imported cases of arbovirus infection pose continued risk to the residents of Hawaii, highlighting the need for rigorous monitoring and surveillance of virus and vectors.

In preparation for future arbovirus introductions, we recommend that Hawaii implement standardized surveillance programs and continue to build vector control infrastructure targeting Ae. aegypti and Ae. albopictus. ${ }^{35}$ Special attention should be given to targeting aquatic habitats of immature $A e$. albopictus, which are often cryptic, and the fine-scale mapping of the occurrence and abundances of Ae. aegypti and $A e$. albopictus, including their relative abundance. Local vector populations should be screened for resistance to first line of defense insecticides, and we suggest that the suitability and acceptability of novel vector control interventions in Hawaii be assessed.

Received October 2, 2019. Accepted for publication November 9, 2019.

Published online February 10, 2020.

Note: Supplemental table appears at www.ajtmh.org.

Acknowledgments: We would like to thank the Hawaii Dengue Response Team for all their efforts in completing this investigation and Jose Ruiz-Valcarcel at the CDC Dengue Branch for his help on the manuscript.

Financial support: Funding was provided by the Division of VectorBorne Infectious Diseases, Centers for Disease Control and Prevention.

Disclaimer: The findings and conclusions in this report are those of the authors and do not necessarily represent the views of the Centers for Disease Control and Prevention.

Authors' addresses: Jeomhee M. Hasty, Environmental Health Services Division, Hawaii Department of Health, Vector Control Branch, Aiea, HI, E-mail: jeomhee.hasty@doh.hawaii.gov. Gilberto E. Felix, Manuel Amador, Roberto Barrera, Stephanie Martinez-Conde, and Ryan R. Hemme, Entomology and Ecology Team, Dengue Branch, Centers for Disease Control and Prevention, San Juan, Puerto Rico, E-mails: ckn5@cdc.gov, maa4@ cdc.gov, amz9@cdc.gov, mzn4@cdc.gov, and rhemme@cdc.gov. Gillberto S. Santiago, Jorge L. Munoz-Jordan, and Freddy A. Medina, Surveillance and Research Laboratory Team, Dengue Branch, Centers for Disease Control and Prevention, San Juan, Puerto Rico, E-mails: fbz3@cdc.gov, ckq2@cdc.gov, and fkt3@cdc.gov. Lynn Nakasone, Environmental Health Services Division, Hawaii Department of Health, Aiea, HI, E-mail: lynn.nakasone@doh.hawaii.gov. Sarah Y. Park, Disease Outbreak Control Division, Hawaii Department of Health, Honolulu, HI, E-mail: sarah.park@ doh.hawaii.gov. Steven Okoji and Maricia Save, Environmental Health Services Division, Hawaii Department of Health, Kealakekua, HI, E-mails: steven.okoji@doh.hawaii.gov and maricia.save@doh.hawaii.gov. Eric Honda, Environmental Health Services Division, Hawaii Department of Health, Hilo, HI, E-mail: eric.honda@doh.hawaii.gov. Bernard Asuncion, Environmental Health Services Division, Hawaii Department of Health, Vector Control Branch, Hilo, HI, E-mail: bemard.asuncion@doh.hawaii.gov. Stephen H. Waterman, Dengue Branch, Centers for Disease Control and Prevention, San Juan, Puerto Rico, E-mail: shw2@cdc.gov. Lyle R. Petersen, Division of Vector-Borne Diseases, Centers for Disease Control and Prevention, Fort Collins, CO, E-mail: Ixp2@cdc.gov. David I. Johnston, Disease Outbreak Control Division, Department of Health, Honolulu, HI, E-mail: david.johnston@doh.hawaii.gov.

This is an open-access article distributed under the terms of the Creative Commons Attribution (CC-BY) License, which permits unrestricted use, distribution, and reproduction in any medium, provided the original author and source are credited.

\section{REFERENCES}

1. Bhatt $S$ et al., 2013. The global distribution and burden of dengue. Nature 496: 504-507.

2. Gubler DJ, 2011. Dengue, urbanization and globalization: the unholy trinity of the 21(st) century. Trop Med Health 39: 3-11.

3. Johnston D, Viray M, Ushiroda J, Whelen AC, Sciulli R, Gose R, Lee R, Honda E, Park SY; Hawaii Dengue Response Team, 2016. Notes from the field: outbreak of locally acquired cases of dengue fever-Hawaii, 2015. MMWR Morb Mortal Wkly Rep 65: 34-35.

4. Hawaii Department of Health, 2016. Dengue Outbreak 2015-16. Available at: http://health.hawaii.gov/docd/dengue-outbreak2015/. Accessed January 23, 2018.

5. Gilbertson WE, 1945. Sanitary aspects of the control of the 1943-1944 epidemic of dengue fever in Honolulu. Am J Public Health Nations Health 35: 261-270.

6. Gubler DJ, 1998. Dengue and dengue hemorrhagic fever: its history and resurgence as a global health problem. Gubler DJ, Kuni G, eds. Dengue and Dengue Hemorrhagic fever. London, United Kingdom: CAB International, 1-22.

7. Effler PV et al., 2005. Dengue fever, Hawaii, 2001-2002. Emerg Infect Dis 11: 742-749.

8. Usinger RL, 1944. Entomological phases of the recent dengue epidemic in Honolulu. Public Health Rep 59: 423-430.

9. Wilson GW, 1904. Epidemic of dengue in the territory of Hawaii during 1903. Public Health Rep 19: 67-70.

10. Winchester JC, Kapan DD, 2013. History of Aedes mosquitoes in Hawaii. J Am Mosq Control Assoc 29: 154-163.

11. Van Dine DL, 1904. Mosquitoes in Hawaii. Honolulu, HI: United States Department of Agriculture, 1-30.

12. Wilbar CL Jr., 1947. Control of dengue in Hawaii. Am J Public Health Nations Health 37: 663-674.

13. Wells CJ (US Department of Health, Education, and Welfare, National Communicable Disease Center), 1968. Analysis of Aedes aegypti Eradication Program: State of Hawaii. Atlanta, GA: CDC.

14. Hayes JM et al., 2006. Risk factors for infection during a dengue1 outbreak in Maui, Hawaii, 2001. Trans R Soc Trop Med Hyg 100: 559-566.

15. Hess AD (US Department of Health, Education, and Welfare, Communicable Disease Center), 1957. A Preliminary Appraisal of the Mosquito Control Program in the Territory of Hawaii. Atlanta, GA: CDC, 1-70.

16. Schliessmann DJ, 1967. Initiation of the Aedes aegypti eradication programme of the USA. Bull World Health Organ 36: 604-609.

17. Lambrechts L, Scott TW, Gubler DJ, 2010. Consequences of the expanding global distribution of Aedes albopictus for dengue virus transmission. PLoS Negl Trop Dis 4: e646.

18. Carvalho FD, Moreira LA, 2017. Why is Aedes aegypti Linnaeus so successful as a species? Neotrop Entomol 46: 243-255.

19. Harrington LC, Edman JD, Scott TW, 2001. Why do female Aedes aegypti (Diptera: Culicidae) feed preferentially and frequently on human blood? J Med Entomol 38: 411-422. 
20. Tempelis $\mathrm{CH}$, Hayes RO, Hess AD, Reeves WC, 1970. Bloodfeeding habits of four species of mosquito found in Hawaii. Am J Trop Med Hyg 19: 335-341.

21. Hawley WA, 1988. The biology of Aedes albopictus. J Am Mosq Control Assoc 4: 1-39.

22. Barrera R, Mackay AJ, Amador M, 2013. An improved trap to capture adult container-inhabiting mosquitoes. J Am Mosq Control Assoc 29: 358-368.

23. Santiago GA, Vergne E, Quiles Y, Cosme J, Vazquez J, Medina JF, Medina F, Colon C, Margolis H, Munoz-Jordan JL, 2013. Analytical and clinical performance of the CDC real time RT-PCR assay for detection and typing of dengue virus. PLoS Neg/ Trop Dis 7: e2311.

24. Biggerstaff BJ, 2009. PooledInRate, Version 4.0: An Exce/® AddIn to Compute Infection Rates from Pooled Data. Fort Collins, Colorado: Centers for Disease Control and Prevention.

25. Issack MI, Pursem VN, Barkham TMS, Ng LC, Inoue M, Manraj SS, 2010. Reemergence of dengue in Mauritius. Emerg Infect Dis 16: $716-718$.

26. Zhang FC et al., 2014. Severe dengue outbreak in Yunnan, China, 2013. Int J Infect Dis 27: 4-6.

27. Feldstein LR, Brownstein JS, Brady OJ, Hay SI, Johansson MA, 2015. Dengue on islands: a Bayesian approach to understanding the global ecology of dengue viruses. Trans $R$ Soc Trop Med Hyg 109: 303-312.

28. Kobayashi D, 2018. Dengue virus infection in Aedes albopictus during the 2014 autochthonous dengue outbreak in Tokyo metropolis, Japan. Am J Trop Med Hyg 98: 1460-1468.
29. Kutsuna $S$ et al., 2015. Autochthonous dengue fever, Tokyo, Japan, 2014. Emerg Infect Dis 21: 517-520.

30. Luo L, Jiang LY, Xiao XC, Di B, Jing QL, Wang SY, Tang JL, Wang M, Tang XP, Yang ZC, 2017. The dengue preface to endemic in mainland China: the historical largest outbreak by Aedes albopictus in Guangzhou, 2014. Infect Dis Poverty 6: 148.

31. Reiter $P$ et al., 2003. Texas lifestyle limits transmission of dengue virus. Emerg Infect Dis 9: 86-89.

32. United States Census Bureau, 2012. Population and housing unit counts CPH-2-13, Hawaii. Bureau USC, ed. 2010 Census of Population and Housing. Washington, DC: U.S. Government Priniting Office.

33. Hasty JM, Yang P, Oshiro P, Nakasone L, Whelen C, 2015. Mosquito surveillance program using ovitraps detected Aedes aegypti at the Honolulu International Airport in 2012. Proc Hawaii Entomol Soc 47: 1-11.

34. Hawaii Department of Transportation, 2019. The State of Hawaii Airport Activity Statistics by Calendar Year. Honolulu, HI: Hawaii Department of Transportation, Airports Planning Office, Honolulu International Airport.

35. Centers for Disease Control and Prevention, 2017. Surveillance and Control of Aedes aegypti and Aedes albopictus in the United States. Available at: https://www.cdc.gov/chikungunya/ pdfs/surveillance-and-control-of-aedes-aegypti-and-aedesalbopictus-us.pdf. Accessed April 23, 2019. 